\section{The origin of the}

\section{elements of life}

M.G. Edmunds

Department of Physics and Astronomy, Cardiff University, Wales

$A^{\text {bout } 10 \% \text { of your body weight is hydrogen. The simple }}$ $A_{\text {proton nuclei of the hydrogen have not been altered since }}$ they formed in the Big Bang some 14 billion years ago. But $60 \%$ of you is oxygen, $20 \%$ is carbon and $3-5 \%$ is nitrogen, elements that have been made in nuclear reactions since that distant strange beginning of the Universe. In this article I will try to set out what we know of how and where these elements - so crucial to life as we know it - were formed. We must look at not only the details of the nuclear reactions inside stars, but also at the mechanisms which will deliver these nuclei into the Galaxy, and how their abundances will have built up over time.

We'll start with oxygen. This is the heaviest of the three CNO elements, but the one whose site of birth is probably best understood. Most of the oxygen in the present-day Universe was produced in nuclear reactions just before or during the spectacular stellar explosions known as Type II supernovae. These occur at the end of the life of stars that began with about eight times or more mass than the Sun. The amount of energy that can flow through a star, from its nuclear generation in the star's hot core and up to the surface, is strongly dependent on the mass of the star. A star that is ten times the mass of the Sun has perhaps a thousand times the solar flow of energy. So even though a more massive star has proportionally greater reserves of nuclear fuel than the Sun, it squanders its inherited hydrogen at a prodigious rate, converting it to helium in a well-known cycle of quasi-catalytic nuclear reactions that we shall later look at in detail. The result is a very short lifetime - only a few million years - before an inexorable and rapid sequence of structural changes occurs. As the hydrogen fuel is exhausted deep in the star's core, the core's density and temperature increase under gravitational contraction. Other reactions become possible as the higher relative speeds overcome the electrostatic coulomb barrier repulsion of higher charged nuclei and tunnel through to reaction. The most obvious reaction might seem to be

$$
{ }^{4} \mathrm{He}+{ }^{4} \mathrm{He} \rightarrow{ }^{8} \mathrm{Be}+\gamma
$$

but it has long been known that the beryllium 8 is unstable, decaying back almost immediately to two helium nuclei. Only if a third helium strikes the beryllium before it decays can onward reaction occur to form carbon:

$$
{ }^{8} \mathrm{Be}+{ }^{4} \mathrm{He} \rightarrow{ }^{12} \mathrm{C}+\gamma ;
$$

giving what is known as the "triple-alpha" reaction, because the overall result has been the fusion of three alpha particles. The rate that this reaction must have to explain the lifetimes of certain types of star led to the famous prediction (in the early 1950's) by the late Sir Fred Hoyle that the second reaction must be resonant. And indeed subsequent laboratory measurements did find the required excited energy level in the ${ }^{12} \mathrm{C}$ nucleus, inspiring the Nobel Prize-winner Willy Fowler into a lifetime's work in nuclear astrophysics. The carbon can react onwards by the addition of another alpha particle to give oxygen:

$$
{ }^{12} \mathrm{C}+{ }^{4} \mathrm{He} \rightarrow{ }^{16} \mathrm{O}+\gamma
$$

Surprisingly, the rate of this reaction is still rather poorly known - as discussed in a recent article by Rauscher and Thielemann in this journal. The problem is the very small reaction cross-section at the energies relevant for stellar reactions, which makes it impossibly slow to perform direct laboratory determinations at these energies. Extensive indirect research has taken place in the last thirty years to try and pin it down - since the rate is crucial in determining the relative abundances of the $\mathrm{C}$ and $\mathrm{O}$ nuclei produced in the "burning" of helium. References to the best current estimates are given in Imbriani et al. 2001.

Having used up most of the helium in its core, a massive star will continue through a series of heavier nuclear fuels, each yielding less and less energy despite increasing temperature and pressure. Quite soon the core is no longer able to adjust its pressure balance quickly enough, and its evolution changes from being almost hydrostatic to hydrodynamic - collapsing inwards under the influence of gravitation while temperatures and reaction rates rise steeply. Explosion results from a sudden stiffening of the equation of state in the core as nuclear densities are reached, causing an outward-propagating shock wave that is helped along by the push of neutrinos. Further nucleosynthesis occurs as this shock wave passes, and disrupts the star in a Type II supernova explosion. The enriched material exploding out at tens of thousands of kilometres per second into the interstellar material will deliver the freshly synthesised oxygen for incorporation into future generations of stars and planets. But even before the explosion occurs, considerable material will have been lost from the star by a less extreme, but still quite energetic, stellar "wind". Such winds probably provide the major delivery mechanism for the carbon created in the triple-alpha mechanism, but which has escaped further processing to oxygen.

As outline above, massive stars $\left(\mathrm{M}>8 \mathrm{M}_{\odot}\right)$ are the best candidates for the main source of carbon and oxygen. We now look at the mechanism by which nitrogen is formed. Recall that we mentioned the quasi-catalytic chain of reactions that convert hydrogen into helium. The main chain results from successive

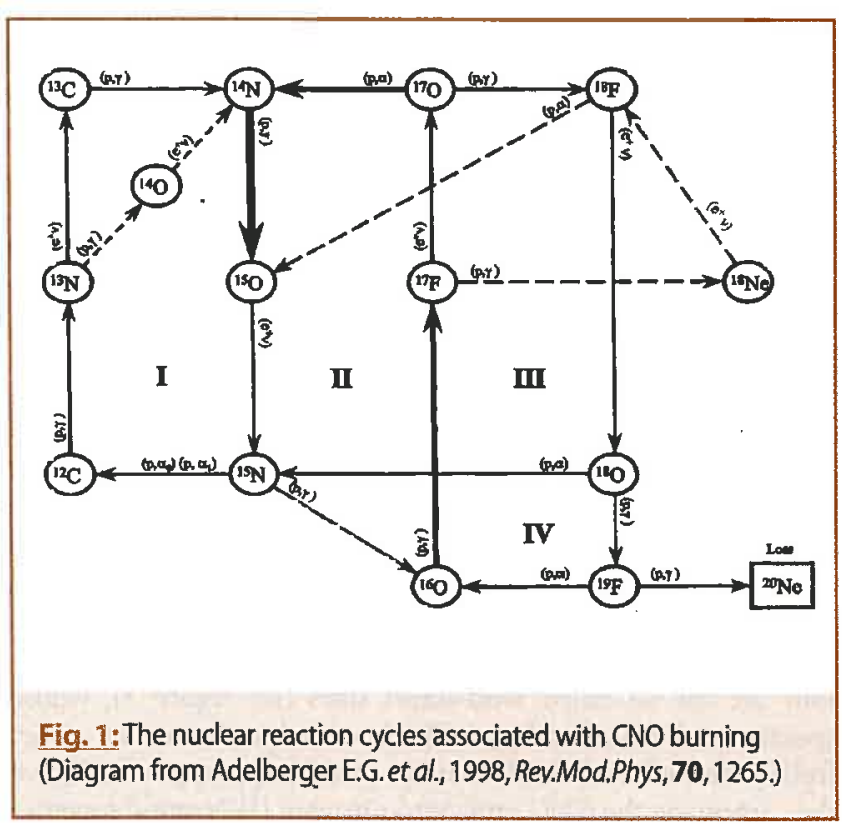


proton captures and beta decays. Suppose we start with the main isotope of carbon, ${ }^{12} \mathrm{C}$, then:

$$
\begin{gathered}
{ }^{12} \mathrm{C}+\mathrm{p} \rightarrow{ }^{13} \mathrm{~N}+\gamma ;{ }^{13} \mathrm{~N} \rightarrow{ }^{13} \mathrm{C}+\mathrm{e}^{+}+v ;{ }^{13} \mathrm{C}+\mathrm{p} \rightarrow{ }^{14} \mathrm{~N}+\gamma \\
{ }^{14} \mathrm{~N}+\mathrm{p} \rightarrow{ }^{15} \mathrm{O}+\gamma ;{ }^{15} \mathrm{O} \rightarrow{ }^{15} \mathrm{~N}+\mathrm{e}^{+}+v ;{ }^{15} \mathrm{~N}+\mathrm{p} \rightarrow{ }^{12} \mathrm{C}+{ }^{4} \mathrm{He}
\end{gathered}
$$

The net result of which is the conversion of four protons into a helium nuclei. The positrons produced will quickly annihilate in the dense plasma. There is a (much slower) side chain feeding oxygen into the cycle at ${ }^{14} \mathrm{~N}$ :

$$
{ }^{16} \mathrm{O}+\mathrm{p} \rightarrow{ }^{17} \mathrm{Ne}+\gamma ;{ }^{17} \mathrm{Ne} \rightarrow{ }^{17} \mathrm{O}+\mathrm{e}^{+}+\mathrm{v} ;{ }^{17} \mathrm{O}+\mathrm{P} \rightarrow{ }^{14} \mathrm{~N}+{ }^{4} \mathrm{He}
$$

Now in the main chain, it is the ${ }^{14} \mathrm{~N}(\mathrm{p}, \gamma)^{15} \mathrm{O}$ which is by far the slowest reaction. This acts as a "bottleneck" - rather like repair works on a road causing the traffic to back up - with the result that the abundance of ${ }^{14} \mathrm{~N}$ builds up. So although the reaction chains are "catalytic" in the sense that the sum of the abundances by number of the nuclei $\mathrm{C}, \mathrm{N}$ and $\mathrm{O}$ is conserved, the actual result is essentially the conversion of ${ }^{12} \mathrm{C}$ (quickly) and ${ }^{16} \mathrm{O}$ (more slowly) into ${ }^{14} \mathrm{~N}$. Not surprisingly, nature is a bit more complicated than just the single "CNO Cycle" describe above - subsequent work suggested a second (CNO "bi-cycle"), and then a third ("tricycle"!) and fourth, as shown in figure 1, but the original cycle is the dominant mechanism. Rather surprisingly one of the reaction rates in the main chains here - the ${ }^{17} \mathrm{O}(\mathrm{p}, \alpha){ }^{14} \mathrm{~N}$ capture - was in error in the literature for many years. I recently tried very elementary calculations of the evolution of element ratios in the chains and kept finding large over-prediction of the ${ }^{17} \mathrm{O} /{ }^{16} \mathrm{O}$ ratio - and it was with some relief that I found more modern determinations (Angulo et al. 1999) show that the old reaction rate was nearly two orders of magnitude too low - with the new (but still uncertain!) rate suitably reducing the ${ }^{17} \mathrm{O}$ abundance. The expected equilibrium element and isotope ratios will be a function of the stellar interior conditions of pressure and temperature. Typical predicted equilibrium element ratios for $\mathrm{N} / \mathrm{O}$ and $\mathrm{N} / \mathrm{C}$ are 10,50 - quite different from the solar system values of $1 / 10,1 / 4$ - indicating that although these chains may be the source of our nitrogen, only a fraction of the carbon and oxygen in the universe has been processed in this way. The prediction of the ${ }^{12} \mathrm{C} /{ }^{13} \mathrm{C}$ isotope ratio is also interesting - CNO cycling tends towards giving a ratio of 3 to 4 , very much lower than the solar system 90, but low values around 6 to 10 are indeed observed in the atmospheres of some giant stars where CNO processed material has been mixed up to change the atmospheric composition, the mixing being caused by convective motions inside the star.

We now have the basic nuclear processes. But the identification of where they occur, or rather of which type of site dominates the supply of the particular element, continues to cause controversy. Massive stars are certainly involved for both oxygen and carbon. Both helium "burning" alpha-capture reactions, and CNO cycles of proton capture will occur at shell-like regions with the appropriate conditions within the star. Towards the end of their brief lives very strong winds of gas are driven off the surfaces of massive stars, reducing their mass and revealing the layers deep inside the star where the products of the recent nuclear reactions can be seen. The winds deliver the synthesised material into interstellar space. Particularly good illustrations of this behaviour are the so-called Wolf-Rayet stars (see figure 2), whose spectra and classification indicate overabundances of either freshly formed carbon (WC stars) or subsequent processing of the carbon via the CNO cycle into nitrogen (WN stars) together with very strong gas outflows. These (quite rare) stars represent a short stage in a massive star's life, which we just happen to have glimpsed. Extensive stellar evolution computations (e.g. Maeder 1992) can follow these changes, and indicate how much new carbon, nitrogen and oxygen is released to the interstellar medium in the stellar winds before the even more spectacular release in supernovae

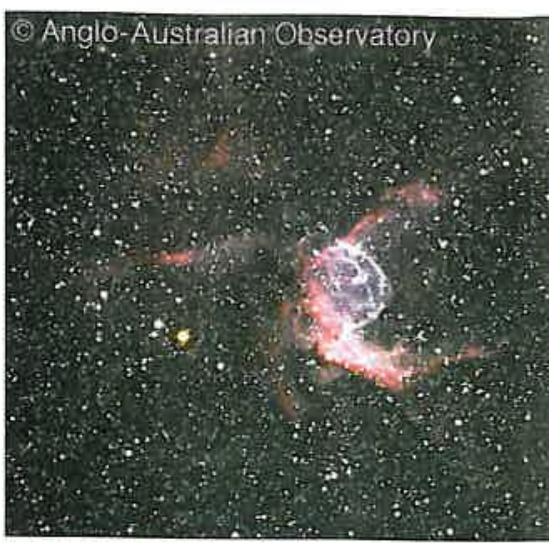

Fig. 2: A Wolf-Rayet star with its nebula including stellar wind gas with newly synthesis carbon and nitrogen. (Photo from AngloAustralian Observatory) explosion at the end of the stars' lives. The release is shown by Andre Madaer's diagram in figure 3, a picture that is now some years old but still remains the best that I know. To identify the sites that dominate CNO nucleosynthesis, our own efforts (Henry, Edmunds \& Köppen 2000) used the results of such calculations, and also of models of the evolution of rather lower (in fact "intermediate" 2-8 Mœ) mass stars. Lower mass stars might be important because, although individually they cannot contribute as much as a more massive star, there are many more of them. Star formation favours the small. Their element production can again be by $\mathrm{CNO}$ processing to produce nitrogen (from the $\mathrm{C}$ or $\mathrm{O}$ seed) and - in their later life as giant stars - alpha-capture reactions. Our method is to look at the chemical composition of a wide range of systems - both stars in our own Galaxy, and gaseous nebulae in a wide range of other galaxies. It is then possible to plot diagrams of the observed carbon to oxygen ( $\mathrm{C} / \mathrm{O}$, see figure 4 ) and nitrogen to oxygen $(\mathrm{N} / \mathrm{O}$, see figure 5 ) ratios as a function of the

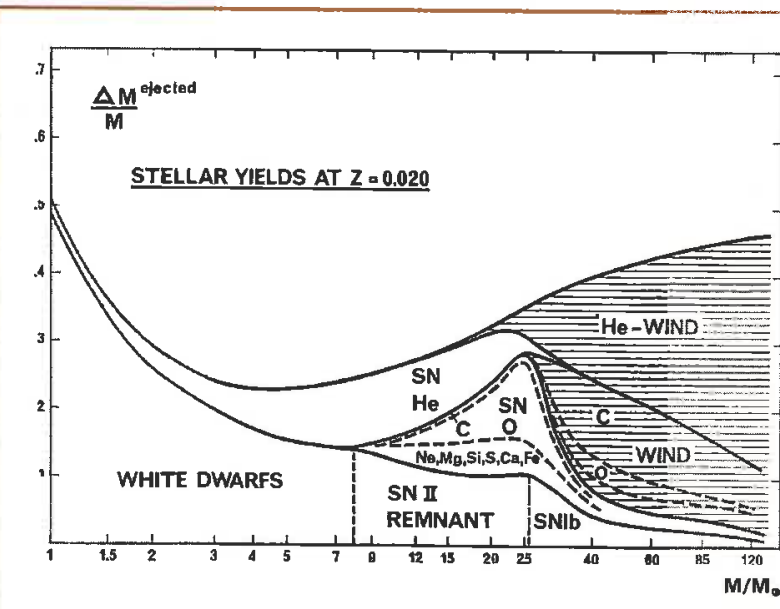

Fig. 3: Schematic diagram by Maeder (1992) illustrating the zones of newly synthesised material released from massive stars into the interstellar material. The vertical axis is the ejected mass fraction (or remnant in the lower part), and the hatched areas show the strong winds. The calculations assumed the stars initially had the same elemental composition as the Sun. 
oxygen to hydrogen $(\mathrm{O} / \mathrm{H})$ ratio in these objects. Note that we are now dealing with number rather than mass ratios of element abundance. The $\mathrm{O} / \mathrm{H}$ ratio acts as a good indicator of the general amount of star formation, stellar evolution and consequent synthesis of heavy elements that has gone on in a system. The bulk of this oxygen originated in Type II supernova explosion of massive stars. The systematics of these diagrams, combined with the stellar nucleosynthesis predictions, allow us to have a pretty good guess at the dominant sources. Obviously we also have to fold the relative birthrate numbers of stars of different masses into the calculations.

The $\mathrm{C} / \mathrm{O}$ diagram suggests that relatively more carbon is produced at low oxygen abundances than at high - otherwise the trend should have been horizontal on the figure. This may well be related to the fact that stellar winds are more intense in stars with high oxygen abundances (probably due to the details of the wind generation mechanism). Quantitative agreement with massive star calculations (c.f. Maeder op cit) implies that it is indeed massive stars (of order 8 solar masses or more) that contribute most of the carbon. This is despite the know existence of less massive stars which do show overabundances (by up to 100 times) of carbon in their atmospheres. The overabundant carbon must have come from internal nucleosynthesis, but these so-called "carbon stars" are not contributing the bulk of the interstellar carbon. Indeed, the nomenclature "carbon star" is rather misleading since carbon is still a fairly minor constituent of such stars, compared to hydrogen or helium. But once the ratio of carbon-to-oxygen exceeds unity in a stellar atmosphere it has a radical and very noticeable effect of the spectrum of the star. The oxygen atoms can no longer tie up all the carbon atoms in carbon monoxide molecules, and the spectra of $\mathrm{C}_{2}$ and other carbon-based molecules dominate the spectrum. It is still unknown exactly how much of the carbon from either massive or lower mass stars is delivered to the interstellar medium in solid form - the carbon vapour can condense (in certain conditions) in the stellar atmospheres. This is a very important problem in the study of the origin and evolution of interstellar dust in galaxies.

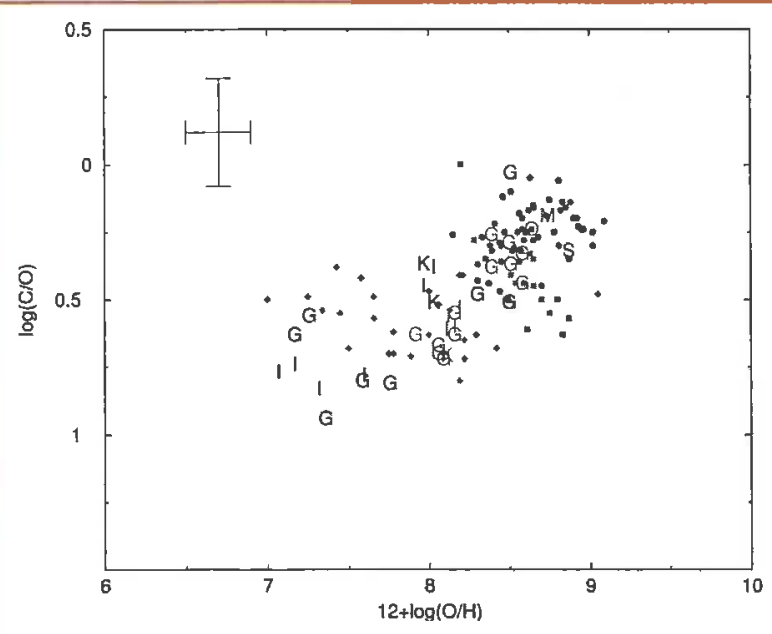

Fig. 4: Logarithmic plot of observed carbon-to-oxygen ratios $(\mathrm{C} / \mathrm{O})$ against oxygen-to-hydrogen ratio $(\mathrm{O} / \mathrm{H})$ for gaseous nebulae (i.e. essentially the interstellar material) in our own and other galaxies, together with some stars. The details of the nomenclature and sources can be found in Henry, R.C.B.r Edmunds, M.G. and Köppen, J. 2000.

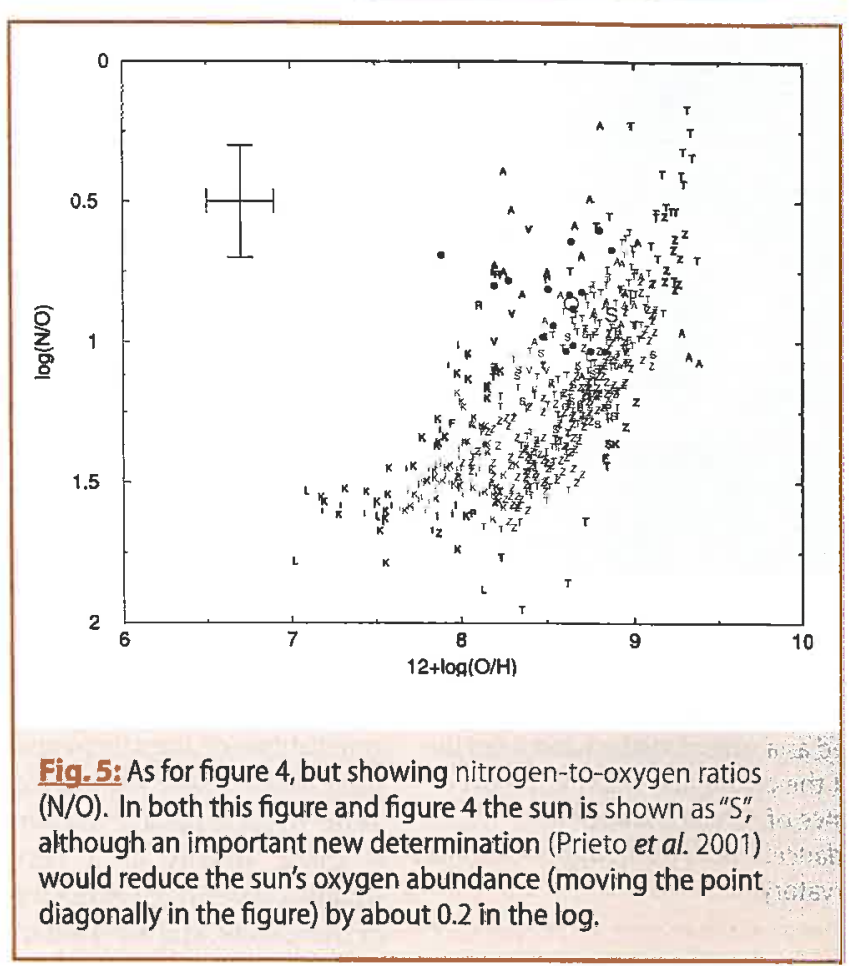

For nitrogen the situation is reversed - it is the intermediate mass stars (perhaps 2 to $6 \mathrm{M}_{\odot}$ ) which are the main contributors. We should introduce a subtle complication here, based on where the carbon and oxygen that is $\mathrm{CNO}$ processed into nitrogen comes from. If the carbon and oxygen is that which came into the star when it formed out the interstellar material, then the nitrogen produced is said to be "secondary" - since it was produced out of pre-existing seed nuclei. Elementary models for the chemical evolution of galaxies then predict that the $\mathrm{N} / \mathrm{O}$ ratio will increase linearly with increasing $\mathrm{O} / \mathrm{H}$. If the star itself produces the carbon and oxygen by helium burning, and then that material is subsequently CNO processed, then the nitrogen produced is said to be "primary". In this case we would expect that the N/O ratio would be constant with increasing $\mathrm{O} / \mathrm{H}$, except perhaps for some small effects due to abundance-induced changes in stellar structure or stellar winds. The appearance of the $\mathrm{N} / \mathrm{O}$ versus $\mathrm{O} / \mathrm{H}$ diagram indicates that the nitrogen has a primary source at low oxygen abundance, but a dominating secondary source at higher abundances. Indeed at higher abundances the behaviour is even steeper than the expected linear behaviour, probably due to the aforementioned abundance effect on overall stellar evolution. Computational models of the so-called "intermediate" mass stars between about 2 and 8 solar masses indicate (e.g. van den Hoek and Groenenwegen 1997) sufficient production (or "yield") to account for the observed nitrogen abundances. Clear evidence of material in which $\mathrm{C}$ and $\mathrm{O}$ have been processed into $\mathrm{N}$ can be seen in the spectra of planetary nebulae. These planetary nebulae (see figure 6) are the glowing gas thrown off by intermediate mass stars after the red giant stage of their life, as they settle down to fade away as white dwarfs. It is not yet finally resolved whether massive stars might make a significant contribution in systems with very low element abundances. This is important because low abundances represent the very earliest stage of galactic evolution. If only the intermediate mass stars contribute, the delivery of nitrogen to the interstellar medium lags behind the rapid $\left(10^{6}\right.$ years) massive stars' contribution of oxygen. The $\mathrm{N} / \mathrm{O}$ ratio can then be used as a kind of "age" indicator until the few $10^{8}$ years 


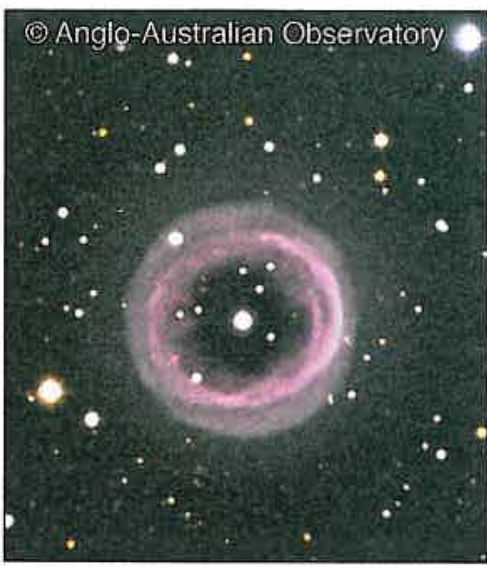

Fig.6: A planetary nebulae. The name "planetary" originates historically with their disk-like appearance in primitive telescopes. The nebula gas has been ejected as a violent stellar wind from the star at the centre, and will often show evidence of enhance nitrogen abundance. (Photo from Anglo-Australian Observatory) later when the intermediate star production begins to be released. Recent calculations (Cheffi et al. 2001) suggest that even the very first generation of $4-8$ solar mass stars with no pre-existing heavy elements may be able to produce carbon and subsequently process it to nitrogen. Whether even more massive stars can do the same and be a significant source of nitrogen remains to be seen.

Overall we now have a picture in which the relative amount of oxygen in the interstellar medium builds up with time as successive generations of stars form and their massive stars explode as Type II supernovae. Carbon is made mainly at a very slightly earlier stage in the life of these stars, and released in strong stellar winds. Nitrogen comes mainly from intermediate mass stars. Over time, nitrogen becomes relatively more abundant compared to oxygen, while carbon relatively declines slightly. It is interesting to speculate at what point in this build up there are sufficient materials to produce life. Of course, the necessary conditions for the formation of stable planets will probably have to be reached first - and for this iron and silicon seem necessary, at least on our current (and probably rather naive!) views.

Almost all theories of star formation agree that the first generation will have contained - and perhaps have been dominated by stars above a few solar masses. So the basic building blocks of life - carbon, nitrogen and oxygen - will have been present in small quantities from within a million years or so of the birth of very first stars. We ourselves are the archaeological remains of nuclear reactions that occurred from the earliest stars right up until 4.6 billion years ago when the formation of the solar system effectively cut us off from the interstellar medium.

\section{References}

Angulo C., et al. 1999, Nucl.Phys A656, 3. Available at http://pntpm.uib.ac.be/nacre.htm

Chieffi, A., Dominguez, I., Limongi, M. and Straniero, O. 2001, ApJ. 554, 1159.

Henry, R.C.B., Edmunds, M.G. and Köppen, J. 2000, ApJ. 541, 660.

Imbriani, G. et al. ApJ., 558, 903.

Maeder A. 1992, Astron.Astrophys., 264, 105.

Prieto, C.A., Lambert, D.L. and Asplund, M. 2000, ApJ. 556, L63, 2001

Rauscher \& Thielemann 2001 Europhysics News ****

van den Hoek, L.B. and Groenewegen, M.A.T. 1997, Astron.Astrophys. Supp. 123, 305.

\section{General References}

Cowley C.R. An Introduction to Cosmochemistry, Cambridge University Press, 1995

Pagel, B.E.J. Nucleosynthesis and the Chemical Evolution of Galaxies Cambridge University Press, 1997

Rolfs C.E. and Rodney W.S. Cauldrons in the Cosmos, University of Chicago Press, 1988. 